01;03;09

\title{
Моделирование лазерного энергоподвода в газовый поток
}

\author{
(C) Т.А. Киселева ${ }^{1,2}$, Т.А. Коротаева ${ }^{1}$, В.И. Яковлев ${ }^{1}$ \\ ${ }^{1}$ Институт теоретической и прикладной механики им. С.А. Христиановича СО РАН, \\ Новосибирск, Россия \\ ${ }^{2}$ Новосибирский национальный исследовательский государственный университет, \\ Новосибирск, Россия \\ E-mail: bobarykina@ngs.ru
}

Поступило в Редакцию 15 ноября 2018 г.

В окончательной редакции 15 ноября 2018 г.

Принято к публикации 28 декабря 2018 г.

\begin{abstract}
Представлены результаты численного моделирования лазерного энергоподвода в сверхзвуковой поток. В отличие от общепринятого подхода в новой расчетной модели учитывается не только поглощенная мощность, но и импульс плазмы пробоя в области фокусировки излучения. Величина импульса определяется в рамках модели поглощения излучения за фронтом светодетонационной волны. Представлен анализ полученных результатов.
\end{abstract}

DOI: 10.21883/PJTF.2019.07.47533.17592

Лазерное излучение наряду с другими источниками энергии используется в аэрофизических исследованиях возможностей управления структурой газовых потоков уже более 20 лет [1-3]. Для интерпретации результатов экспериментов во многих численных исследованиях моделируется объемный источник энергии [4] с пространственно-временны́м распределением энергии (мощности) в виде

$$
E(x, y, z, t)=E(t) \exp \left[-\left(x^{2} / a^{2}+y^{2} / b^{2}+z^{2} / c^{2}\right)\right]
$$

с характерными пространственными масштабами $a, b$ и $c$ и интервалом времени между энергоимпульсами $t=1 / f$, где $f$ - частота следования импульсов излучения. При небольшой длительности импульсов и малом диаметре области пробоя выполняются условия „мгновенности“ и „точечности“ энерговыделения; изобарическая стадия динамики плазмы пробоя соответствует моделям точечного взрыва с учетом или без учета противодавления в зависимости от условий экспериментов [5]. В неподвижном газе расширение плазмы является сферически-симметричным в предельном случае $a, b, c \rightarrow 0$.

При протяженной фокусировке луча и достаточно высокой плотности мощности (зависит от длины волны излучения [6]) поглощение излучения осуществляется в узкой зоне за фронтом светодетонационной волны (СДВ), скорость фронта которой $V$ определяется соотношением

$$
V=\left[2\left(\gamma^{2}-1\right) J / \rho_{0}\right]^{1 / 3},
$$

где $\gamma \approx 1.2, J-$ плотность мощности $\left[\mathrm{W} / \mathrm{m}^{2}\right]$, $\rho_{0}$ - невозмущенная плотность газа. При увеличении плотности мощности на механизм переноса плазменного фронта начинает влиять собственное излучение плазмы пробоя, и эта закономерность претерпевает изменения [7]. В отличие от объемного поглощения наличие высокоскоростного фронта распространения волны поглощения излучения существенно изменяет газодинамику лазерной плазмы. Об этом свидетельствуют полученные методом численного моделирования результаты работы [8], позволившие выделить характерные особенности течения за фронтом СДВ. Можно также предположить, что подобные структурные изменения течения свойственны и другим, более скоростным режимам распространения разрядов.

Выявленные в [8] особенности динамики плазмы лазерного пробоя в неподвижном газе состоят в следующем:

а) за фронтом волны световой детонации формируется высокоскоростное струйное течение плазмы вдоль направления $(O x)$ луча;

b) параметры плазмы на достаточном удалении $x$ от пробоя с хорошим приближением можно определить в предположении изоэнтропического течения, используя

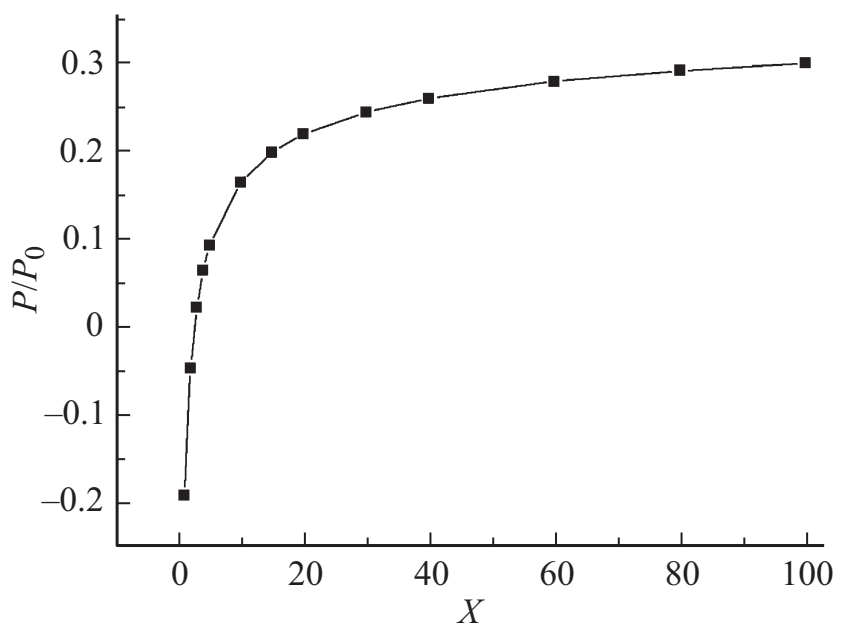

Рис. 1. Изменение импульса плазмы пробоя в зависимости от ее протяженности. 

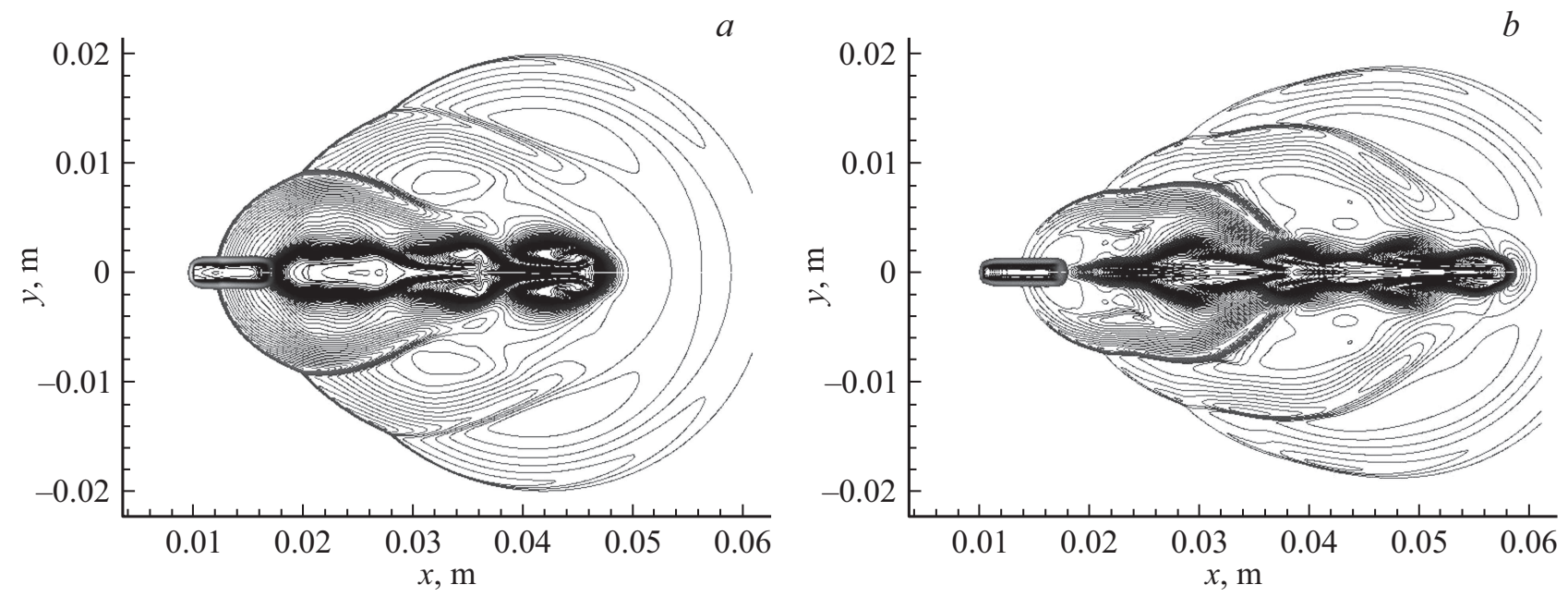

Рис. 2. Распределение плотности после четвертого пробоя газа. Пояснение в тексте.

нестационарное решение для давления в рамках модели точечного взрыва с применением кинематического преобразования $t=x / V$.

Применительно к задаче о влиянии лазерного энергоподвода на поток указанные результаты позволяют сделать важный вывод о том, что кроме повышения температуры и давления плазма лазерного пробоя при определенных условиях может получить также направленную скорость вдоль распространения излучения. Этот дополнительный фактор воздействия лазерного излучения при анализе данных ранее не учитывался, что оправдано при короткой фокусировке луча.

Задача настоящей работы состоит в определении на основе численного моделирования особенностей лазерного энергоподвода в сверхзвуковой поток с учетом помимо поглощенной энергии также импульса, приобретаемого плазмой пробоя при протяженной фокусировке излучения в режиме СДВ. В расчете использовались условия эксперимента $[5,9]$, в котором протяженный энергоисточник в сверхзвуковом потоке аргона (число Маха $\mathrm{M}=2$ ) формировался импульсно-периодическим излучением $\mathrm{CO}_{2}$-лазера. Параметры излучения близки к необходимым для формирования протяженной области пробоя в СДВ-режиме.

Величина импульса плазмы пробоя определяется в рамках упрощенной модели: одновременно с „мгновенным“ энерговыделением в области каустики луча плазма пробоя приобретает импульс $P$ в направлении распространения излучения. Его величину можно рассчитать с использованием представленных в [8] аналитических решений, определяющих распределения плотности $\rho(x)$, скорости $u(x)$, радиуса $r(x)$ струйного течения. Приобретенный импульс $P$ плазмы пробоя составляет величину $P / P_{0}=\left(1 / x_{0}\right) \int \rho(x) u(x)\left[r(x) / r_{0}\right]^{2} d x$, где $P_{0}=\rho_{0} V$, $u$ - скорость струи в расчетной системе координат, пределы интегрирования $x=0-x_{0}$, где $x_{0}=V \tau-$ расстояние, которое фронт СДВ проходит за время им- пульса $\tau$ излучения, т. е. протяженность плазмы пробоя. Введение фактора $\left[r(x) / r_{0}\right]^{2}\left(r_{0}-\right.$ радиус каустики) учитывает увеличение площади сечения струи по мере удаления от фронта. Результаты расчета относительной величины импульса $P / P_{0}$ в зависимости от относительного расстояния $X=x / d\left(d=2 r_{0}\right)$ представлены на рис. 1. Импульс плазмы пробоя интенсивно растет при малых $X$, слабо увеличиваясь при $X>15$ до значений в диапазоне $P / P_{0} \approx 0.2-0.3$. Отметим, что режим СДВ формируется при $X \gg 1$. Величина импульса зависит от условий фокусировки с учетом определенной взаимосвязи параметров: $V \propto J^{1 / 3}$ и $J \propto d^{-2}$.

Численное моделирование лазерного энергоподвода в сверхзвуковой поток аргона проведено с использованием академической версии пакета программ ANSYS FLUENT для нестационарной задачи в осесимметричной постановке. Трапециевидная область решения, покрытая сеткой с 300000 четырехугольных ячеек, включала в себя подобласть, вытянутую в направлении потока. Эта подобласть содержит источники энергии и силы, задаваемые с помощью написанных на языке программирования Си функций (,user defined functions“"). В рамках используемого пакета программ решались уравнения Эйлера. Применялись „density-based“ решатель FLUENT и неявная схема Рое второго порядка точности аппроксимации. В качестве граничных условий на внешней границе расчетной области использовался тип „pressure-far-field“, т.е. задавались статическое давление $p=0.56 \cdot 10^{5} \mathrm{~J} / \mathrm{m}^{3}$, плотность $\rho_{0}=2.3 \mathrm{~kg} / \mathrm{m}^{3}$ и число Маха $\mathrm{M}=2$, на входной границе использовалось граничное условие „pressure-inlet“. На выходной границе ставилось условие типа „pressure-outlet". В уравнении состояния задавались опции „ideal gas“ с постоянной теплоемкостью.

Для источника энергии учитывалась удельная поглощенная мощность импульса $\left[\mathrm{W} / \mathrm{m}^{3}\right]$; в уравнении сохранения количества движения применялись расчетные 

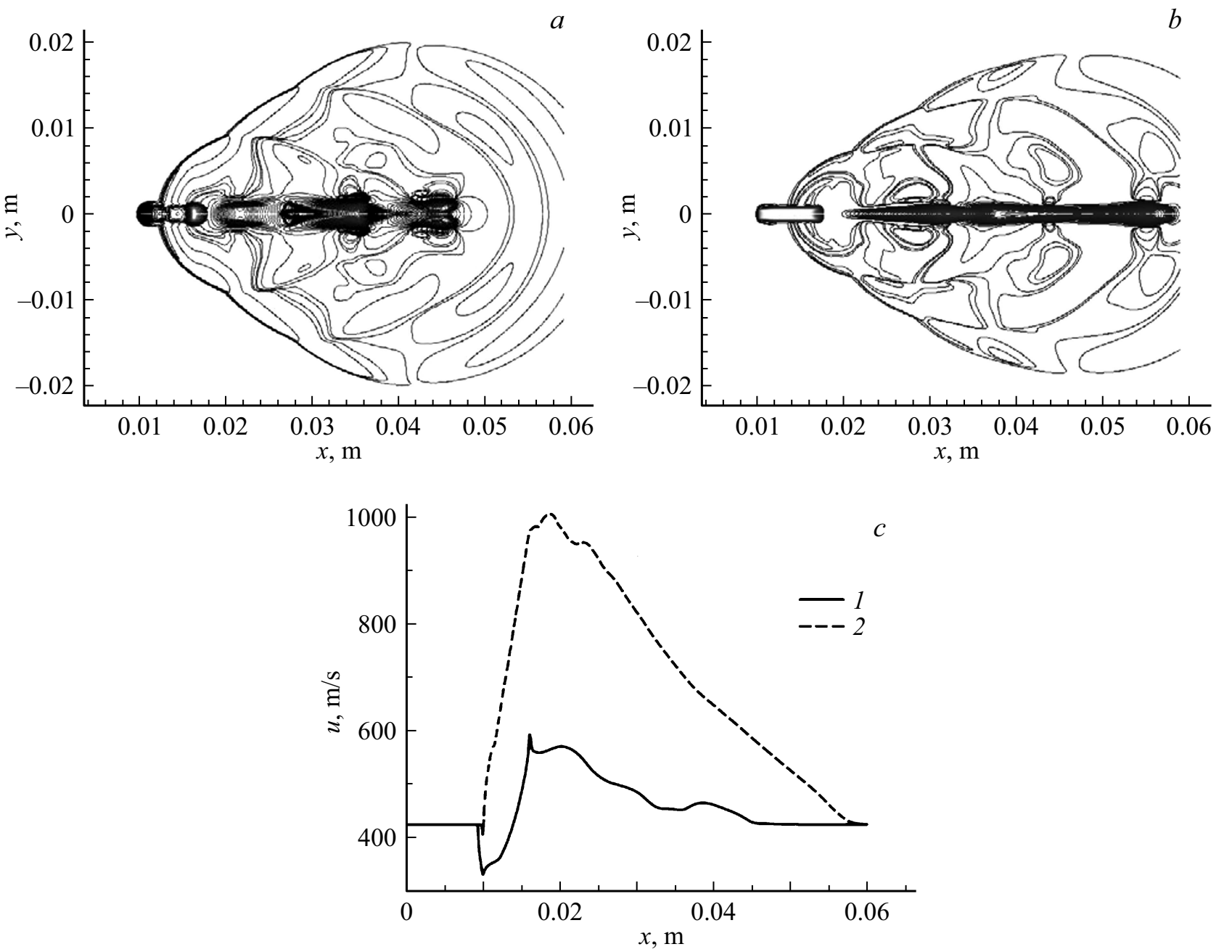

Рис. 3. Распределение скорости вдоль оси потока. 1 - подвод энергии, 2 - подвод энергии с учетом импульса плазмы пробоя. Пояснение в тексте.

значения плотности импульса $P / \tau\left[\mathrm{N} / \mathrm{m}^{3}\right]$. В экспериментах с частотой $45 \mathrm{kHz}$ энергия и длительность импульса излучения были следующими: $E=40 \mathrm{~mJ}$ и $\tau=1 \mu \mathrm{s}$. Объем области энергоподвода определялся исходя из его диаметра $d=0.2 \mathrm{~mm}$ и протяженности области пробоя $(6 \mathrm{~mm})$. Использовались значения $J=1.27 \cdot 10^{12} \mathrm{~W} / \mathrm{m}^{2}$ и $P / P_{0}=0.25(X \approx 30)$ в соответствии с данными рис. 1 .

На рис. 2 представлены результаты расчета осевых распределений плотности в потоке с учетом подвода только энергии $(a)$ и энергии и импульса $P$ плазмы пробоя $(b)$ в момент времени после четвертого пробоя. В первом случае происходит последовательное относительно слабое увеличение области низкой плотности при каждом подводе энергии, в то время как во втором сильное увеличение (расширение) происходит на начальной стадии (второй пробой) при последующем относительно слабом изменении размеров этой области. На рис. 3 представлены результаты осевого распределения скорости потока. В отличие от сильных перепадов значений скорости за областью подвода энергии (рис. 3,a) при дополнительном учете импульса плазмы в области пробоя распределение скорости по оси не претерпевает сильных изменений (рис. 3,b). Происходит формирование струйного течения с более высокой скоростью струи $(1000 \mathrm{~m} / \mathrm{s}$, диаметр $\sim 2 \mathrm{~mm})$ по сравнению со скоростью окружающего потока. Это также подтверждают результаты осредненных по времени счета распределений скорости вдоль оси потока $(y=0)$, представленные на рис. 3, .

Таким образом, показано, что лазерный энергоподвод в режиме высокоскоростного распространения разряда (в частности, СДВ) приводит к значительным изменениям структуры сверхзвукового течения за областью подвода энергии. Использование данного режима может расширить возможности применения лазерного излучения при решении задач управления скоростными течениями.

Работа выполнена при финансовой поддержке Российского фонда фундаментальных исследований (гранты РФФИ № 18-08-00449 и 16-08-00526). 


\section{Список литературы}

[1] Зудов В.Н., Третьяков П.К. // ЖТФ. 2018. Т. 88. В. 3. С. 350357.

[2] Борзов Ю.В., Михайлов В.М., Рыбка И.В., Савищенко Н.П., Юрьев А.С. // Инж.-физ. журн. 1994. Т. 66. № 5. С. 515-520.

[3] Бобарыкина Т.А., Малов А.Н., Оришич А.М., Чиркашенко В.Ф., Яковлев В.И. // Квантовая электроника. 2014. Т. 44. № 9. C. 836-840.

[4] Гувернюк С.В., Самойлов А.Б. // Письма в ЖТФ. 1997. Т. 23. B. 9. C. 1-9.

[5] Фомин В.М., Яковлев В.И. Энергообмен в сверхзвуковых газоплазменных течениях с ударными волнами. М.: ФИЗМАТЛИТ, 2017. 368 с.

[6] Райзер Ю.П. Основы современной физики газоразрядных процессов. М.: Наука, 1980. 416 с.

[7] Фишер В.И., Хараш В.М. // ЖЭТФ. 1982. Т. 83. В. 5. C. $1738-1746$.

[8] Thomas P.D. // AIAA J. 1977. V. 15. N 10. P. 1405-1409.

[9] Зудов В.Н., Третьяков П.К., Тупикин А.В., Яковлев В.И. // Изв. РАН. Механика жидкости и газа. 2003. Т. 38. № 5. C. $140-153$. 